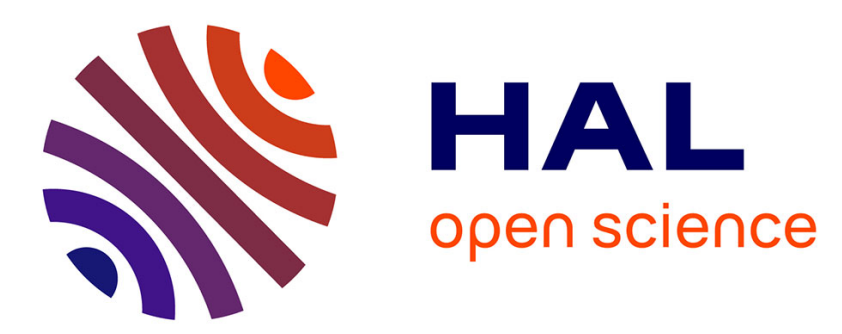

\title{
Instabilities in passive dispersion oscillating fiber ring cavities
}

François Copie, Matteo Conforti, Alexandre Kudlinski, Arnaud Mussot, Fabio Biancalana, Stefano Trillo

\section{To cite this version:}

François Copie, Matteo Conforti, Alexandre Kudlinski, Arnaud Mussot, Fabio Biancalana, et al.. Instabilities in passive dispersion oscillating fiber ring cavities. The European Physical Journal D: Atomic, molecular, optical and plasma physics, 2017, 71 (5), 10.1140/epjd/e2017-80130-x . hal02386245

\section{HAL Id: hal-02386245 \\ https://hal.science/hal-02386245}

Submitted on 29 Nov 2019

HAL is a multi-disciplinary open access archive for the deposit and dissemination of scientific research documents, whether they are published or not. The documents may come from teaching and research institutions in France or abroad, or from public or private research centers.
L'archive ouverte pluridisciplinaire HAL, est destinée au dépôt et à la diffusion de documents scientifiques de niveau recherche, publiés ou non, émanant des établissements d'enseignement et de recherche français ou étrangers, des laboratoires publics ou privés. 


\title{
Instabilities in passive dispersion oscillating fiber ring cavities
}

\author{
François Copie ${ }^{\mathrm{a}, 1}$, Matteo Conforti ${ }^{\mathrm{b}, 1}$, Alexandre Kudlinski ${ }^{\mathrm{c}, 1}$, Arnaud \\ Mussot $^{\mathrm{e}, 1}$, Fabio Biancalana ${ }^{\mathrm{d}, 2}$, Stefano Trillo ${ }^{\mathrm{f}, 3}$ \\ ${ }^{1}$ Univ. Lille, CNRS, UMR 8523 - PhLAM - Physique des Lasers Atomes et Molécules, IRCICA, F-59000 Lille, France \\ ${ }^{2}$ School of Engineering and Physical Sciences, Heriot-Watt University, EH14 4AS Edinburgh, UK \\ ${ }^{3}$ Department of Engineering, University of Ferrara, Via Saragat 1, 44122 Ferrara, Italy
}

Received: date / Accepted: date

\begin{abstract}
We investigate theoretically and experimentally the development of instabilities in passive ring cavities composed of pieces of fiber with different dispersion. We derive an extended version of the LugiatoLefever equation that permits to model dispersion oscillating cavity and we demonstrate that this equation is valid well beyond the mean field approximation. We review the theory of Turing (modulational) and Faraday (parametric) instability in inhomogeneous fiber. We report the experimental demonstration of the generation of stable Turing and Faraday pattern in the same device, which can be controlled by changing the detuning and/or the input power. Moreover, we experimentally record the round-trip-to-round-trip dynamics of the spectrum, which shows that Turing and Faraday instabilities not only differ by their characteristic frequency but also by their dynamical behavior.
\end{abstract}

Keywords First keyword · Second keyword · More

\section{Introduction}

Modulation instability (MI) is a central mechanism in nonlinear optics at the origin of many complex nonlinear phenomena such as Fermi-Pasta-Ulam recurence [1, 2], Peregrine and Kuznetsov-Ma soliton excitation [3, 4], supercontinum generation $[5,6]$, development of rogue waves $[7,8]$ or frequency combs $[9,10]$. It is ruled by a phase-matched four-photon process leading to the exponential growth of a pair of symmetric side lobes around

\footnotetext{
a e-mail: Francois.Copie@univ-lille1.fr

${ }^{\mathrm{b}}$ e-mail: Matteo.Conforti@univ-lille1.fr

c e-mail: Alexandre.Kudlinski@univ-lille1.fr

de-mail: f.biancalana@hw.ac.uk

e e-mail: Arnaud.Mussot@univ-lille1.fr

fe-mail: trlsfn@unife.it
}

the pump. It has been observed in free running fiber systems in the early 80's [11] and later on exploited to develop large band fiber optical parametric amplifiers for telecommunication applications $[12,13]$, short pulse amplifications [14] or signal processing [15]. In passive cavities, MI is usually referred to as Turing instability by analogy with the pattern formation in chemical experiments [16], and besides its fundamental interest it has a wealth of applications ranging from optical memory [17], self referencing $[18,19]$ to ultra-stable optical clock [18]. Quite recently, the idea of periodically modulating one parameter along fiber length has been investigated in the context of MI in free running configurations [20]. It has been shown that it adds a new degree of freedom to these fiber systems leading to the formation of new quasi-phase matched waves $[21,20,22,23]$. In passive cavities, it can be achieved by periodically modulating group velocity dispersion [24], nonlinearity [25] or losses [26]. It significantly modifies the dynamics of these cavities where, in addition to Turing instabilities observable in uniform cavities, the periodic modulation acts as a forcing term leading to the birth of parametric resonances [27], also known as Faraday instabilities in direct analogy with the seminal discovery of Faraday in a vertically vibrating bowl [28]. In dispersion oscillating cavities, it was first theoretically predicted by some of us [24] and then demonstrated experimentally [29]. We showed for the first time that while Turing and Faraday instabilities have different physical origins, they can exist within the same physical system. Following this first observation in the steady state regime, the round-tripto-round-trip behaviour has been investigated revealing a complex dynamics during the switching from one instability regime to the other [30]. The competition arising between these instabilities was recently investigated theoretically in more details and reveal that in 
addition to have different frequencies, these instabilities are chracterized by a different behavior in the time domain with $\mathrm{P} 1$ or $\mathrm{P} 2$ regimes [31].

The Lugiato-Lefever Equation (LLE) $[32,33]$ is the fundamental tool exploited to study theoretically and numerically nonlinear dynamics in passive cavities. Some of us extended LLE to the description of inhomogeneous cavities, and showed its validity well beyond the assumption (mean-field) under which it has been originally derived [24]. However a satisfactory explanation of why LLE can describe situations that goes beyond the mean field approximation is still missing.

The aim of this paper is threefold. First, we propose an original and rigorous derivation of LLE model under minimal assumptions, which applies both to uniform and dispersion oscillating cavities. Secondly, we review the theory of modulational and parametric instabilities in homoneneous and passive resonators. Lastly, we report our main experimental results obtained in these cavities showing the observation of Turing and Faraday instabilities within the same physical system.

\section{Rigorous derivation of LLE}

We consider the passive fiber ring resonator sketched in Fig. 1(a). The input is a train of equally spaced pulses, perfectly synchronized, i.e. the cavity length is such that after each cavity transit, the recirculating pulse perfectly overlaps in time with the next pulse of the train. The pulses being much longer than the typical MI period, we can consider a continuous wave pumping. The electric field at the coupler is modelled by the Ikeda map [34], describing the cavity boundary conditions:

$$
E^{(n+1)}(Z=0, T)=\theta E_{i n}(T)+\rho e^{i \phi_{0}} E^{(n)}(Z=L, T),
$$

where $Z$ measures the distance along the fiber, $T$ is the retarded time, $E$ and $E_{\text {in }}$ are the intracavity and input electric field envelopes, $\rho^{2}+\theta^{2}=1$ are the reflection and transmission coefficients, $\phi_{0}=\beta_{0} L$ is the linear phase detuning, $\beta_{0}=\omega_{0} n\left(\omega_{0}\right) / c$ is the wavenumber at the laser frequency $\omega_{0}$ and $L$ is the cavity length. $n$ being an integer counting the round-trip number. The electric field envelope evolves inside the cavity $(0 \leq$ $Z \leq L)$ according to the nonlinear Schrödinger equation (NLSE):

$i \frac{\partial E^{(n)}}{\partial Z}-\frac{\beta_{2}(Z)}{2} \frac{\partial^{2} E^{(n)}}{\partial T^{2}}+\gamma\left|E^{(n)}\right|^{2} E^{(n)}=0$,

where $\beta_{2}(Z)$ is the group velocity dispersion (GVD) and $\gamma$ is the nonlinear coefficient of the fiber. The function
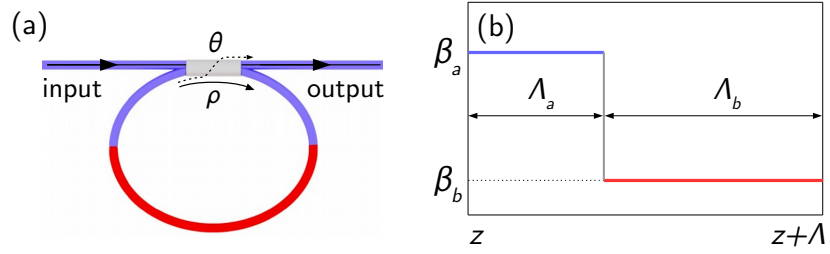

Fig. 1 (a) Schematic illustration of a non-uniform passive fiber ring cavity. (b) Piecewise constant dispersion map over one period of the GVD.

$\beta_{2}(Z)$ is periodic and its period is in general a fraction of the cavity length $L$ [31]. In the rest of the paper we focus on the simplest case where the periodicity of the dispersion is equal to the cavity length. In general both the dispersion and the nonlinearity change, however in our experimental range we can assume that only the GVD changes and $\gamma$ is constant.

The LLE has been originally derived from Eqs. (12 ) in the so called mean-field approximation [32,35], i.e. assuming that the intracavity field does not evolve significantly over a round-trip. This entails several strong constraints: the cavity is short with respect to nonlinear and dispersion length, the intensity loss at the mirror and the cavity detuning are small. However, LLE holds valid even when several of these assumptions are violated. A typical example is the parametric instability in a dispersion oscillating cavity, where the dispersion and the field change rapidly over a single round-trip [36].

In the following, we show that the only assumption needed in order to derive LLE is that only one longitudinal mode of the cavity is excited.

We start by performing a phase rotation

$A^{(n)}(Z, T)=E^{(n)}(Z, T) \exp \left[i \beta_{0} Z\right]$, in order to incorporate the rapid phase variation into NLSE and remove it from the boundary conditions. The equations for the fast variable $A$ are:

$A^{(n+1)}(Z=0, T)=\theta E_{i n}(T)+\rho A^{(n)}(Z=L, T)$,

$i \frac{\partial A^{(n)}}{\partial Z}+\beta_{0} A^{(n)}-\frac{\beta_{2}(Z)}{2} \frac{\partial^{2} A^{(n)}}{\partial T^{2}}+\gamma\left|A^{(n)}\right|^{2} A^{(n)}=0$.

Now we let the spatial variable to assume all values from 0 to infinity, i.e. we unfold the cavity. Even if $Z$ is a continuous variable, the field is physically accessible only at the coupler, i.e. at distances $Z=n L$. We incorporate the boundary conditions in the propagation equation with the aid of the delta function, so that we can write Eqs. (3-4) in the following equivalent compact way:

$\begin{aligned} i \frac{\partial A}{\partial Z}+ & \beta_{0} A-\frac{\beta_{2}(Z)}{2} \frac{\partial^{2} A}{\partial T^{2}}+\gamma|A|^{2} A= \\ & i \sum_{n} \delta(Z-n L)\left[\theta E_{i n}(T)+(\rho-1) A\right] .\end{aligned}$ 
By expanding the delta comb in Fourier series:

$$
\sum_{n} \delta(Z-n L)=\frac{1}{L} \sum_{n} e^{i n \frac{2 \pi}{L} Z}=\frac{1}{L} \sum_{n} e^{i n k Z},
$$

we get

$$
\begin{aligned}
i \frac{\partial A}{\partial Z}+ & \beta_{0} A-\frac{\beta_{2}(Z)}{2} \frac{\partial^{2} A}{\partial T^{2}}+\gamma|A|^{2} A= \\
& \frac{i}{L} \sum_{n} e^{i n k Z}\left[\theta E_{i n}(T)+(\rho-1) A\right] .
\end{aligned}
$$

In order to recognize exponential terms oscillating at the same rate, we come back to the slow variable $E=A \exp \left[-i \beta_{0} Z\right]$ :

$$
\begin{aligned}
i \frac{\partial E}{\partial Z}- & \frac{\beta_{2}(Z)}{2} \frac{\partial^{2} E}{\partial T^{2}}+\gamma|E|^{2} E= \\
& \frac{i \theta}{L} E_{i n} \sum_{n} e^{i\left(n k-\beta_{0}\right) Z}+i \frac{\rho-1}{L} E \sum_{n} e^{i n k Z} .
\end{aligned}
$$

Equation (8) is a NLSE forced by two combs with equal wavenumber spacing $k=2 \pi / L$ and a relative shift $\beta_{0}$. The solution of (8) can be written as a sum of slowly-varying envelopes, which modulates the longitudinal modes of the cavity, as $E(Z, T)=\sum_{n} E_{n}(Z, T) e^{i k n Z}$

It is of fundamental importance to choose the correct forcing terms for each mode, in order to neglect the fast-rotating exponential in favor of the slowly evolving ones. When the intracavity power is reasonably small, we can assume that we have only one mode present in the cavity, i.e.

$E(Z, T)=E_{0}(Z, T)$.

This assumption permits to describe all the phenomena we observe in our experiments and in most of the situations. Only when several longitudinal mode are present in the cavity, as observed recently in a tristable resonator [37], the single LLE ceases to be valid and a more complex model has to be used. It is worth noting that the single longitudinal mode operation is the only hypothesis that we made so far.

We retain only the quasi-phase-matched forcing terms in (8), i.e. the terms that are multiplied by the exponentials with the smallest argument. We find:

$i \frac{\partial E_{0}}{\partial Z}-\frac{\beta_{2}(Z)}{2} \frac{\partial^{2} E_{0}}{\partial T^{2}}+\gamma\left|E_{0}\right|^{2} E_{0}=\frac{i \theta}{L} E_{i n} e^{i \delta Z / L}+i \frac{\rho-1}{L} E_{0}$,

where the cavity detuning $\delta$ is defined as follows

$\delta=m k-\beta_{0}, m=\arg \min _{n}\left|n k-\beta_{0}\right|$,

that implies $\delta \in[-\pi, \pi]$. This fixes the detuning validity range of LLE. Any choice $|\delta|>\pi$ means that we have neglected the slowest oscillating term in favor of a rapid one, which means that we have chosen the wrong $n$ in the first summation in (8). Note that we do not need to assume that the detuning is small.

We can remove the exponential from the pump by the phase rotation $E_{0}(Z, T)=U_{0}(Z, T) \exp [i \delta Z / L]$, to find the LLE in the usual (dimensional) form:

$i \frac{\partial U_{0}}{\partial Z}-\frac{\beta_{2}(Z)}{2} \frac{\partial^{2} U_{0}}{\partial T^{2}}+\gamma\left|U_{0}\right|^{2} U_{0}=\frac{i \theta}{L} E_{i n}+\left(\frac{\delta_{0}}{L}-i \frac{\alpha}{L}\right) U_{0}$

where $\alpha=1-\rho$. The intrinsic fiber losses, that have been neglected up to now, can be accounted for by taking $\alpha$ as the total round-trip losses.

We can write Eq. (12) in a convenient non-dimensional form as follows:

$i \frac{\partial u}{\partial z}-\frac{\beta(z)}{2} \frac{\partial^{2} u}{\partial t^{2}}+|u|^{2} u=(\delta-i \alpha) u+i S$,

where $z=Z / L, t=T / T_{0}, u=U \sqrt{\gamma L}, T_{0}=\sqrt{\left|\beta_{2}^{a v}\right| L}$; $S=\theta u_{i n}(t)$, and $\beta(z)=\beta_{2}(z) /\left|\beta_{2}^{a v}\right|, \beta_{2}^{a v}$ being the average group velocity dispersion. The normalized periodic dispersion profile (normalized period $\Lambda=1$ ) is of the form $\beta(z)=\beta_{a v}+\beta_{m} f(z)$, where $\beta_{m}$ is the amplitude and $f(z)$ the shape of the dispersion modulation.

\section{Linear stability analysis}

The stationary solution of Eq. (13), $u_{0}(z, t)=\sqrt{P_{u}}$, which can be assumed real without loss of generality, follows from the steady state response $P=P\left(P_{u}\right)$, explicitly $P=P_{u}\left[\left(P_{u}-\delta\right)^{2}+\alpha^{2}\right]$, where $P=|S|^{2}$ is the input power and $P_{u}=\left|u_{0}\right|^{2}$ is the intracavity power. The steady state response is bistable whenever $\delta^{2}>3 \alpha^{2}$. In this case the function $P_{u}(P)$ is multivalued in the range $P\left(P_{u}^{+}\right) \leq P \leq P\left(P_{u}^{-}\right)$where $P_{u}^{ \pm}=\left(2 \delta \pm \sqrt{\delta^{2}-3 \alpha^{2}}\right) / 2$ stands for the knees of the bistable response. In the multivalued region, out of the three possible solutions, only the lower and the higher ones are stable. The intermediate one, associated to a negative slope, is dynamically unstable so it is not reachable in the experiments.

The cavity steady states can destabilize through the exponential growth of modulations which can be due to a Turing (modulation instability) or Faraday (parametric instability) mechanism, respectively. The Turing instability is characteristic of a uniform cavity (in a periodic case it is affected only by the average quantities), whereas the Faraday instability is a consequence of the parametric resonance due to the forcing, and hence the characteristics of the instability are affected by the period and the strength of the perturbation. However both 
follow from a linear stability analysis of the steady solution $u_{0}$, which at some point needs to be specialized to describe the two mechanisms. To this end, we start by considering the evolution of a perturbed solution $u(z, t)=\sqrt{P_{u}}+[p(z, t)+i q(z, t)]$, where we assume that the real functions $p, q$ are much smaller than $u_{0}$.

By linearization of Eq. (13), we obtain a linear system ruling the evolution of the perturbation:

$$
\begin{gathered}
\frac{\partial p}{\partial z}-\frac{\beta(z)}{2} \frac{\partial^{2} q}{\partial t^{2}}+\left(P_{u}-\delta\right) q+\alpha p=0, \\
\frac{\partial q}{\partial z}+\frac{\beta(z)}{2} \frac{\partial^{2} p}{\partial t^{2}}-\left(3 P_{u}-\delta\right) p+\alpha q=0 .
\end{gathered}
$$

Taking the Fourier transform in time

$\left[\hat{p}(z, \omega)=\int u(z, t) e^{i \omega t} d t\right]$ of this system leads, for each frequency, to

$$
\frac{d}{d z}\left[\begin{array}{l}
\hat{p} \\
\hat{q}
\end{array}\right]=\left[\begin{array}{cc}
-\alpha & -g(z) \\
h(z) & -\alpha
\end{array}\right]\left[\begin{array}{l}
\hat{p} \\
\hat{q}
\end{array}\right]
$$

where $g(z)=\frac{\beta(z)}{2} \omega^{2}+P_{u}-\delta$ and $h(z)=\frac{\beta(z)}{2} \omega^{2}+3 P_{u}-$ $\delta$.

\subsection{Uniform cavity: Turing instability}

We start by considering a uniform cavity, where $\beta_{m}=$ $0, \beta(z)=\beta_{a v}$. System (16) is similar to a damped harmonic oscillator, whose oscillation spatial frequency (wavenumber) is

$k_{a v}=\sqrt{h_{a v} g_{a v}}=\sqrt{\left(\frac{\beta_{a v}}{2} \omega^{2}+2 P_{u}-\delta\right)^{2}-\left(P_{u}\right)^{2}}$.

Its eigenvalues, which rule the $z$ evolution, read $-\alpha \pm$ $i k_{a v}$. When the detuning $\delta$ is sufficiently high, $k_{a v}$ can become imaginary in a certain range of $\omega$, and the solution of Eqs. (16) involves two exponentials with real argument. In this range, if $\left|k_{a v}\right|>\alpha$ the perturbations $\hat{p}, \hat{q}$ grows exponentially $\propto \exp [G(\omega) z]$ with growth rate $G(\omega)=-\alpha+\sqrt{-h_{a v} g_{a v}}$, entailing MI (Turing) of the stationary solution [35]. The most unstable Turing frequency $\omega=\omega_{T}$ and its corresponding gain, can be easily calculated from the eigenvalues to be

$\omega_{T}=\sqrt{\frac{2}{\beta_{a v}}\left(\delta-2 P_{u}\right)}, \quad G\left(\omega_{T}\right)=P_{u}-\alpha$.

We recall that, unlike the cavityless fiber configuration where MI occurs only in the anomalous GVD regime and without threshold, in the cavity, MI occurs also with normal GVD and has a threshold $P_{u}=\alpha$, obtained by imposing $G=0$ in Eq. (18).
The linear stability analysis presented here allows to determine the conditions of instability with respect to small perturbations but does not provide any information on the dynamics of large amplitude modulated states. In other words, MI is the generating mechanism of the Turing pattern but only in a subset of the unstable region, the growth of the sideband can generate a stable pattern. This has been analyzed in details in [38]. The region of parameter where MI can lead to the generation of a stable Turing pattern is highlighted in green in Fig. 3.

3.2 Periodically modulated cavity: Faraday instability

Before proceeding with the analysis, it is useful to factorize the effect of the losses through the transformation $[\hat{p}, \hat{q}]=[\tilde{p}, \tilde{q}] \exp (-\alpha z)$, that transforms Eqs. (16) into a one degrees of freedom Hamiltonian system with canonical coordinates $[\tilde{p}, \tilde{q}]$ :

$\frac{d}{d z}\left[\begin{array}{l}\tilde{p} \\ \tilde{q}\end{array}\right]=\left[\begin{array}{cc}0 & -g(z) \\ h(z) & 0\end{array}\right]\left[\begin{array}{l}\tilde{p} \\ \tilde{q}\end{array}\right]$

Since the coefficients in the equation are $z$-periodic with period $\Lambda$, Floquet theory applies. This amounts to study the evolution over one period $\Lambda$ ( $\Lambda=1$ in this paper $)$, to obtain the Floquet map $\Phi$ which is the two by two real matrix defined by $[\tilde{p}(\Lambda), \tilde{q}(\Lambda)]^{T}=\Phi[\tilde{p}(0), \tilde{q}(0)]^{T}$. As a result $[\tilde{p}(n \Lambda), \tilde{q}(n \Lambda)]^{T}=\Phi^{n}[\tilde{p}(0), \tilde{q}(0)]^{T}$. Note that $\Phi$ necessarily has determinant one, since it is obtained by integrating a Hamiltonian dynamics, which preserves phase space volume. As a consequence, the two eigenvalues $\lambda^{ \pm}$of $\Phi$ are constrained to lie either both on the unit circle, or both on the real axis. Only in the latter case the system can be unstable, the instability being associated with $|\lambda|>1$ according to Floquet theory.

Since the system (19) is not autonomous, it cannot be solved analytically in general. Nevertheless, the above observations allow us to obtain some information about its stability for relatively small $\beta_{m}$, which, importantly, hold valid regardless of the specific shape of the forcing $f(z)$ [39].

To see this, let us start from the unperturbed limit $\beta_{m}=0, \beta(z)=\beta_{a v}$. It is then straightforward to integrate the system (19). The Floquet map is then given by

$\Phi_{a v}=\left[\begin{array}{cc}\cos \left(k_{a v} \Lambda\right) & -\frac{g_{a v}}{k_{a v}} \sin \left(k_{a v} \Lambda\right) \\ \frac{k_{a v}}{g_{a v}} \sin \left(k_{a v} \Lambda\right) & \cos \left(k_{a v} \Lambda\right)\end{array}\right]$.

The eigenvalues of $\Phi_{a v}$ can be easily computed as

$\lambda_{a v}^{ \pm}=\exp \left( \pm i k_{a v} \Lambda\right)$ 
We assume that $k_{a v}(\omega)$ is real, i.e. the uniform cavity is stable with respect to perturbations at frequency $\omega$, and ask ourselves what happens by switching on the periodic dispersion described by $f(z)$. The system (19) becomes non-autonomous and hence it is no longer possible, in general, to give a simple closed form expression of the eigenvalues. Nevertheless, we do know that, for sufficiently small $\beta_{m}$, the eigenvalues of $\Phi$ must be close to the eigenvalues $\lambda_{a v}^{ \pm}$. We then distinguish two cases:

1. Off-resonant case $k_{a v} \neq \frac{m \pi}{\Lambda}$. Since $k_{a v} \Lambda \neq m \pi$, it follows from Eq. (21) that $\lambda_{a v}^{-}=\left(\lambda_{a v}^{+}\right)^{*}$, are distinct and they both lie on the unit circle, away from the real axis. They then must remain on the unit circle under perturbation since, for the reasons explained above, they cannot move into the complex plane away from the unit circle. In this case, the stationary solution is linearly stable under a sufficiently small perturbation $\beta_{m} f(z)$ and this statement does not depend on the precise form of $f(z)$.

2. On-resonant case $k_{a v}=\frac{m \pi}{\Lambda}$. It follows from Eq. (21) that $\lambda_{a v}^{+}=\lambda_{a v}^{-}= \pm 1$ (upper or lower sign holds for $m$ even or odd, respectively) is a doubly degenerate eigenvalue of $\Phi_{a v}$. Under a small perturbation, the degeneracy can be lifted and two real eigenvalues can be created, one greater than one, one less than one in absolute value. The system has then become unstable. In principle, under very peculiar perturbations, the eigenvalues might also move along the circle implying that the system remain stable. However, for the most common perturbations (sinusoidal, square wave, sawtooth, comb, ...), the system, for some $m$, destabilizes under an arbitrarily small perturbation, following the split of the eigenvalues on the real axis at the degenerate points \pm 1 .

We recap by saying that, when the forcing is switched on, the instability sets in under the resonant condition $k_{a v}=\frac{m \pi}{\Lambda}$. Recalling the expression of $k_{a v}=k_{a v}(\omega)$ in Eq. (17), it is straightforward to show that the $m$-th order resonance is fulfilled at frequency $\omega=\omega_{m}$, where

$$
\omega_{m}=\sqrt{\left\{\frac{2}{\beta_{a v}}\left(\delta-2 P_{u}\right)\right\} \pm\left[\frac{2}{\beta_{a v}} \sqrt{\left(\frac{m \pi}{\Lambda}\right)^{2}+P_{u}^{2}}\right]}
$$

We emphasize that the resonance condition

$$
k_{a v}\left(\omega_{m}\right)=m \frac{\pi}{\Lambda}=m \frac{k_{g}}{2}
$$

is the condition of parametric resonance, i.e. the natural spatial frequency of the unperturbed harmonic oscillator $\left(k_{a v}\right)$ is equal to a multiple of half the forcing spatial frequency $\left(k_{g}=\pi / \Lambda\right)$.

We return now to the original (damped) system for the perturbations $[\hat{p}, \hat{q}]$. The Floquet map is simply given by $\Psi_{a v}=\exp (-\alpha \Lambda) \Phi_{a v}$, whose eigenvalues read as:

$\sigma_{a v}^{ \pm}=\exp (-\alpha \Lambda) \lambda_{a v}^{ \pm}=\exp \left[\left(-\alpha \pm i k_{a v}\right) \Lambda\right]$.

That means that the eigenvalues $\sigma_{a v}^{ \pm}$lie in the complex plane either on a circle of radius $\exp (-\alpha \Lambda)$ (case 1$)$, or on the real axis (case 2). Now the perturbation can grow only if the forcing $\beta_{m}$ is sufficient to push one of the eigenvalues outside the unit circle. In this case, the perturbations $\hat{p}, \hat{q}$ will experience an exponential growth with rate $G(\omega)=\ln \left(\max \left|\sigma^{ \pm}\right|\right) / \Lambda$. This means that there is a threshold on $\beta_{m}$ for the onset of the parametric instability. On the same footing, for a fixed $\beta_{m}$, there exist a power threshold for the parametric instability to appear.

To summarize, the Faraday instability appears in general at multiple frequencies $\omega_{m}$ given by Eq. (22) which depend on the period of the forcing and represent the tips of the unstable regions known as Arnold tongues, whereas a threshold in the strength of the forcing (or intracavity power) exists which depends on the losses (the higher the losses, the higher the threshold) and on the specific shape of the perturbation (though no general analytical formulas can be given for the threshold).

The stability analysis presented here allows to determine the conditions of parametric instability with respect to small perturbations but does not provide any information on the dynamics of large amplitude modulated states. In other words, parametric instability is the generating mechanism of the Faraday pattern but only in a subset of the unstable region the growth of the sideband can generate a stable pattern. The region where parametric instability can lead to the generation of a stable Faraday pattern is highlighted in blue in Fig. 3 , and it was calculated from numerical simulation of LLE. Above this region, the Faraday pattern becomes unstable and the behavior of the cavity chaotic.

\subsection{Piecewise constant dispersion}

An example of practical interest where the Floquet analysis can be performed analytically is a cavity with a piecewise constant dispersion [36]. This case corresponds to the experimental set-up we used, where the intracavity loop is made of two pieces of different fibers spliced together. The fiber-ring thus exhibit a step-like longitudinal dispersion profile (see Fig. 1(b)). The Floquet 

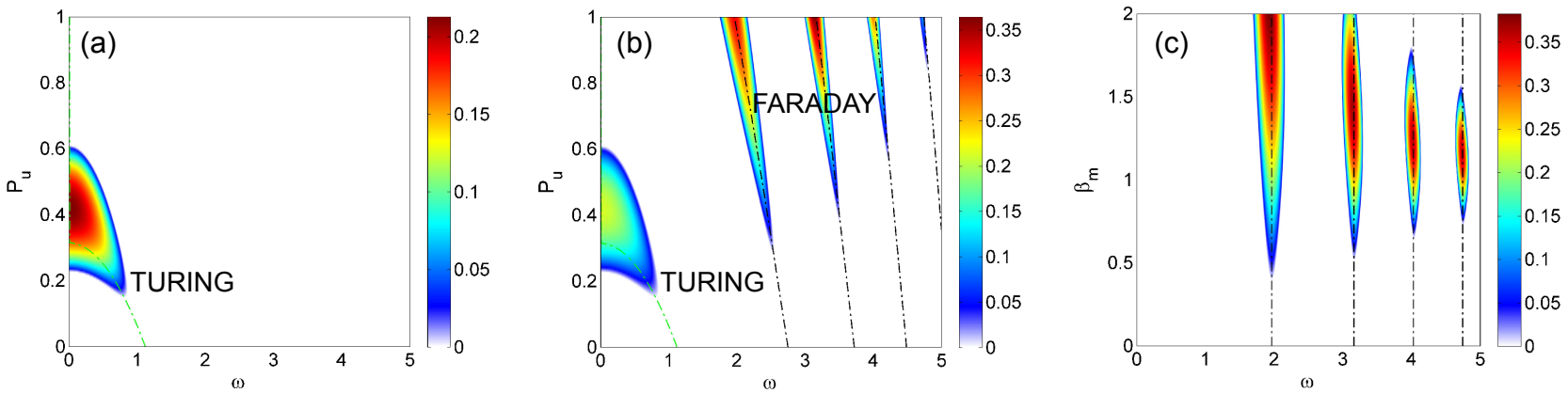

Fig. 2 Color level plot of growth rate $G(\omega)$. (a) Uniform cavity $\beta_{a v}=1, \beta_{m}=0, \Lambda=1$. The dashed green curve indicates the peak gain calculated from Eq. (18). (b) Modulated cavity $\beta_{a v}=1, \beta_{a, b}=1 \pm 1.5, \Lambda_{a}=\Lambda_{b}=0.5$. The dashed black curves indicates the peak gain calculated from Eq. (22) for $m=1,2,3,4$. (c) Modulated cavity $\beta_{a v}=1, P_{u}=1, \Lambda_{a}=\Lambda_{b}=0.5$. In all three plots, $\delta=\pi / 5, \alpha=0.15$.

map is given by

$\Psi=\exp (-\alpha \Lambda) \Phi_{a} \Phi_{b}$

where $\Phi_{a, b}$ has the expression (20) calculated for a dispersion $\beta(z)=\beta_{a, b}$, where the two pieces of fiber has length $\Lambda_{a, b}$, such that $\Lambda_{a}+\Lambda_{b}=\Lambda$ and the average dispersion is $\beta_{a v}=\left(\beta_{a} \Lambda_{a}+\beta_{b} \Lambda_{b}\right) / \Lambda$.

The eigenvalues of $\Psi$ are given by

$\sigma^{ \pm}=\frac{D}{2} \pm \sqrt{\frac{D^{2}}{4}-W}$,

where

$$
\begin{aligned}
D=e^{-\alpha \Lambda}\left[2 \cos \left(k_{a} \Lambda_{a}\right) \cos \left(k_{b} \Lambda_{b}\right)-\right. \\
\left.\quad \frac{g_{a} h_{b}+g_{b} h_{a}}{k_{a} k_{b}} \sin \left(k_{a} \Lambda_{a}\right) \sin \left(k_{b} \Lambda_{b}\right)\right],
\end{aligned}
$$

and $W=e^{-2 \alpha \Lambda}$. We have parametric instability if $|D|>(1+W)$, with gain $G(\omega)=\ln \left(\max \left|\sigma^{ \pm}\right|\right) / \Lambda$. In Fig. 2 we report some examples of analytically calculated instability gain. Figure 2(a) shows the gain as a function of perturbation frequency and intracavity power for a homogeneous cavity. In this case, we observe a branch located around zero frequency, that can generate a stable Turing pattern. Figure 2(b) shows the gain for a modulated cavity in the same operating conditions. The Turing branch survives, and we see the generation of several branches due to the periodic forcing. This parametric instability branches (Arnold tongues) are the generating mechanism for the Faraday patterns. The existence of both Turing and Faraday branches in the same device allows us to observe the competition between the two phenomena. Figure 2(c) shows the Arnold tongues as a function of the forcing amplitude $\beta_{m}$ for a fixed power. Due to nonzero losses $\alpha$, there exist a threshold for the onset of the instability (as discussed above), which is generally different for each tongue.

\subsection{Chart of instabilities}

The behavior of the system is controlled by two main parameters, namely power and normalized detuning $\Delta=$ $\delta / \alpha$, which are easily accessible experimentally. It can be summarized in Fig. 3 which shows the domains of the different instabilities in the parameter plane $\left(\Delta, P_{u}\right)$ for a uniform cavity (Fig. 3 (b)), and for a dispersion modulated cavity (Fig. 3(d)) when the average dispersion is normal $\left(\beta_{2}^{a v}>0\right)$. For better clarity, the corresponding steady state responses are also reported in Figs. 3(a) and (c), respectively for a large value of detuning of $\Delta=6.25$ because it allows to capture all the richness of the setup with the excitation of both regimes of instability. Note that it corresponds to the detuning value used in the experiments described below. For $\Delta \geq \sqrt{3}$, the cavity is bistable $[32,35]$, and exhibits an unstable negative slope branch for $P_{u}^{-}<P_{u}<P_{u}^{+}$, where $P_{u}^{ \pm}(\Delta)$ stands for the bistability knees, delimiting the domain labeled "inaccessible" in Fig. 3(b) and (d). Below such domain, the green area corresponds to the region where temporally modulated Turing structures can be excited in uniform and modulated cavities (Fig. 3(b) and (d), respectivelly). This region has been computed numerically and corresponds to the tiny domain where Turing structures, which bifurcate subcritically, can be spontaneously formed [40]. We emphasize that this regime requires to drive the cavity with a detuning $\Delta>4.25$, and with powers belonging to a small portion of the lower branch of the bistable response (highlighted in green over the bistable curve for $\Delta=6.25$ in Fig. 3(a) and (c)). It is important to emphasize that this regime only depends on the average GVD and not on its peri- 
odic modulation. On the contrary, Faraday structures only develop when the cavity is driven over the upper branch and the periodic longitudinal variations are effective.
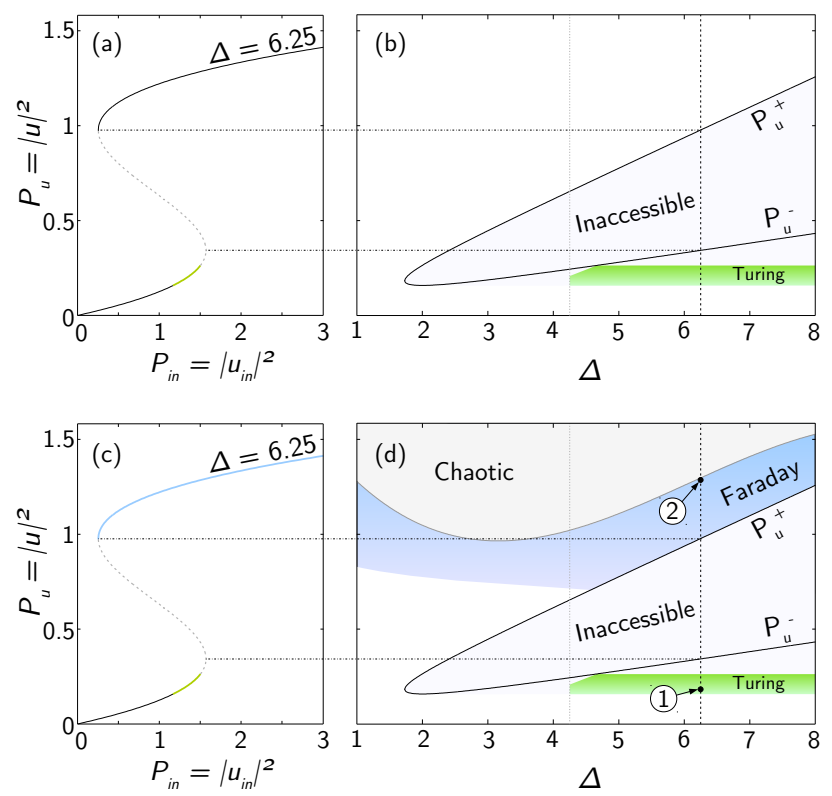

Fig. 3 (a) Normalized steady-state curve for a detuning value $\Delta=6.25$. (b) Instability domains of a uniform cavity in the plane $\left(\Delta, P_{u}\right)$ in the average normal dispersion regime $\left(\beta_{2}^{a v}>0\right)$. The "Inaccesible" region corresponds to the negative-slope branch of the steady-state of (a). The green domain represent the region where Turing modulated structures can be excited. (c-d) Same as (a-b) except that the periodic dispersion is switched on. The Faraday domain of instability appears on the upper branch (for higher powers the Faraday structures destabilize giving rise to chaotic spatiotemporal evolutions). Parameters: $\Lambda_{a}=0.97, \Lambda_{b}=0.03$, $\beta_{a}=1.5, \beta_{b}=-14, \alpha=0.157, \theta^{2}=0.1, \Lambda=1$ : the period of the GVD equals the length of the resonator.

As a result, the stable excitation of Faraday structures requires to operate in the blue domain of Fig. 3(d). Note that no blue area exists in Fig. 3(b) which summarize the behavior of uniform cavities. In that configuration, the upper branch is modulationally stable and thus there is no pattern formation. At higher powers, such Faraday structures destabilize leading to chaotic states (see upper portion of Fig. 3(d)). Note that the Faraday branch (unlike the Turing one) extends also to the monostable regime $\Delta<\sqrt{3}$. However, we choose to focus on the bistable regime where the two instabilities can compete thereby drastically changing the bistable switching dynamics. Indeed, at high detunings $(\Delta>4.25)$, both instability regimes can be excited in the same cavity and switching between Turing and Faraday structures can be controlled by the power (vertical axis in Fig.3(d)).

\section{Experimental results}

\subsection{Steady state regime}

We built a fiber ring cavity presenting the piecewise constant dispersion profile shown in Fig. 4(b). The ring has a total length of $51.6 \mathrm{~m}$, and is composed of a $50 \mathrm{~m}$ long, specially designed dispersion shifted fiber (DSF, with GVD $\beta_{2}=2 \mathrm{ps}^{2} / \mathrm{km}$ at $1550 \mathrm{~nm}$ ) directly spliced to the two pigtails (total length $1.6 \mathrm{~m}$ ) of the input/output coupler made of a standard single-mode fiber (SMF-28 with GVD $\beta_{2}=-19 \mathrm{ps}^{2} / \mathrm{km}$ ). The average nonlinear coefficient is $\gamma=5.5 / \mathrm{W} / \mathrm{km}$ and we numerically checked that the slight difference (inferior to $10 \%$ ) between the nonlinear coefficients does not affect the behaviour of the system. Thus, we can consider that the nonlinear coefficient is almost constant over all the cavity length. The cavity is pumped at $1550 \mathrm{~nm}$ (well below the average zero dispersion wavelength of 1562 $\mathrm{nm}$ ), where the values of GVD reported above gives a normal average dispersion $\beta_{2}^{a v} \approx 1.35 \mathrm{ps}^{2} / \mathrm{km}$. We estimated the finesse to be $\approx 20$. The experimental setup is sketched in Fig. 4(a). In order to operate at constant detuning $\delta$ we use a fraction of the power of the pump called the "Control beam", launched inside the cavity in the direction opposed that of the "Nonlinear beam". We control the linear phase accumulation of light during a round-trip by finely tuning the wavelength of the laser. The output of the "Control beam" is launched into a servo controlled system (PID) to be compared to a reference level related to the desired detuning. It then generates an error signal that finely tunes the pump wavelength to compensate for the environmental fluctuations, and thus locks the value of the detuning. The maximum duration of the locking is strongly linked to the environment fluctuations for they eventually lead to a failure of the PID system. As an example, the evolution of the optical path length of the cavity over $10 \mathrm{~s}$ is shown in Fig. 4 (d). As can be seen, the length of the cavity is stabilized with an accuracy of at least $\lambda / 200$ rms.

In order to validate the general behaviour depicted in Fig. 3(d), we performed experiments with $\Delta=6.25$ where both instabilities can be excited by simply tuning the pump power as predicted in Fig. 3 (d).

Figure 5 shows the results obtained for this detuning. For input powers below $3.9 \mathrm{~W}$ we do not observe any spectral signature of periodic structures in the output spectrum. Indeed the system is stable and simply follows the lower branch of the steady-state response shown in Fig. 5(a). However, when the power exceeds a first MI threshold the system enters the Turing region and exhibits the stable formation of sidebands over 


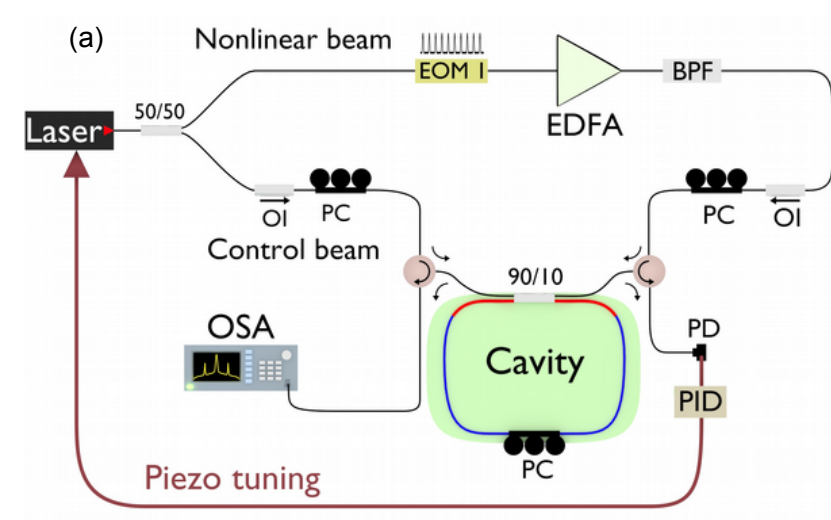

(b)

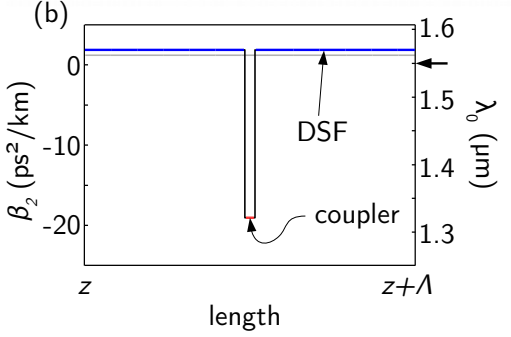

(c)
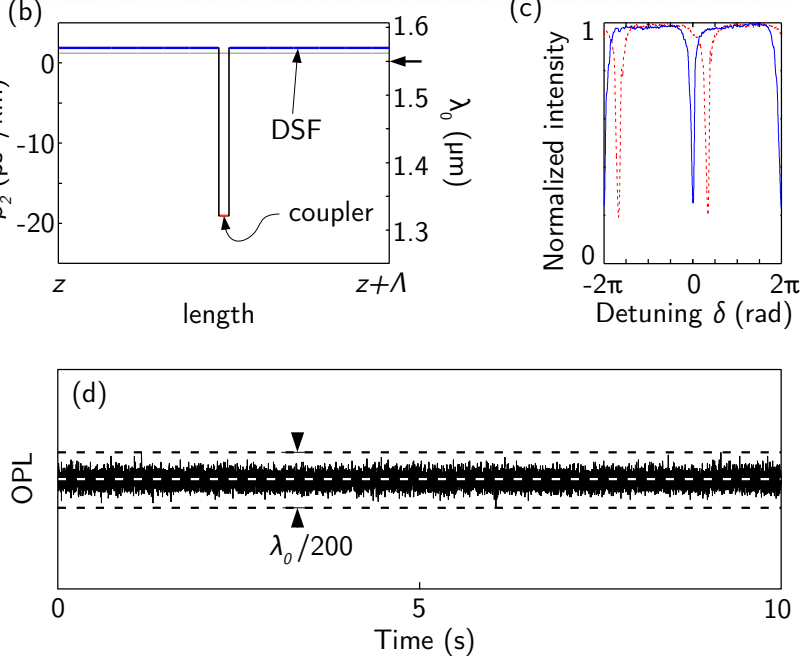

Fig. 4 (a) Experimental setup. (b) GVD map of the cavity over one round-trip, centered on the 90/10 SMF coupler. The gray horizontal line gives the average GVD $\beta_{2}^{a v}$ at the pump wavelength of $1550 \mathrm{~nm}$ (arrow on the right vertical axis, calibrated in terms of wavelengths). (c) Normalized transfer functions of the cavity for the Control beam (dashed red) and the Nonlinear beam in the linear regime (blue). (d) Optical path length (OPL) variation over 10 seconds when the feedback loop is operating.

the lower branch. An example is shown in Fig. 5(d), where the primary sidebands are located at $0.70 \mathrm{THz}$. This is consistent with the fact that, while the periodic solutions corresponding to Turing structures continue to bifurcate sub-critically, a stable branch exists for a finite range of input powers, as shown by the green curve in Fig. 5(a). Then, when the power exceeds the value where the Turing branch merge on the stationary response, the Turing instability induces up-switching towards the upper branch. As described above, however, this branch presents narrowband Faraday instability [see Fig. 5(b)] and hence two sidebands are still observed in the spectra [see Fig. 5(b)], though at much larger frequency $(1.16 \mathrm{THz})$. As can be seen, experimental spectra (blue curves) in Fig. 5(c) and 5(d) are in excellent agreement with numerical simulations (dashed red curves) and with the analytical predictions of the positions for the sidebands (vertical grey lines, $0.69 \mathrm{THz}$ and $1.15 \mathrm{THz}$ respectively). The large difference of frequency shifts between Fig. 5(c) and 5(d) allows us to claim that we have unambiguously observed the crossover between the two instabilities. Thus, we can conclude that the dynamics of this bistable system is dramatically affected by the excitation of modulated structures due to competing Turing and Faraday branches. We showed that by tuning the pump power launched inside the cavity, one can control the dynamics this bistable system by switching from one regime of instability to the other.

\subsection{Transient regime}

In the previous section, spectra corresponding to steady state regimes were recorded by means of an optical spectrum analyser. They are averaged over thousand of round-trips, since the typical integration time of an OSA is of the order of the second, to be compared with a round-trip time that is of the order of the microsecond. The recording of instantaneous spectra has attracted a lot of attention in recent years through the development of real time spectroscopy techniques such as the time-stretch dispersive Fourier transformation (DFT). Its ultra-short recording time enables the recording of shot-to-shot spectral fluctuations associated to ultrafast nonlinear phenomena such as MI [41,42] or supercontinuum generation [43] or round-trip-to-roundtrip dynamics of both passive and active fiber cavities [44-47]. We propose to take benefit of this technique to observe the transient evolution of Turing and Faraday instabilities to capture the growth and saturation of the power of the different unstable bands as well as the switching dynamics between these instabilities driven by abruptly changing the input pump power. The experimental setup shown in Fig. 6 is very similar to that described in the previous section to investigate the steady state behavior of these cavities (Fig. 4(a)), except three details. (i) We add a second EOM (EOM 2) to create either periodic bursts of pump pulses to observe the growth of Turing or Faraday instabilities or a two-level pump pattern to observe the switch from one to the other. (ii) The output spectra of the cavity are recorded by using an OSA (steady state regime) and/or a fast oscilloscope (OSC) coupled to a dispersion compensating fiber (DCF) using the real-time spectroscopy technique (see Ref. [48] for more details). Note that the duration of the pulses is slightly shorter in that case (400 ps) to be able to achieve an accurate frequency to time conversion via the DFT technique. (iii) The length 


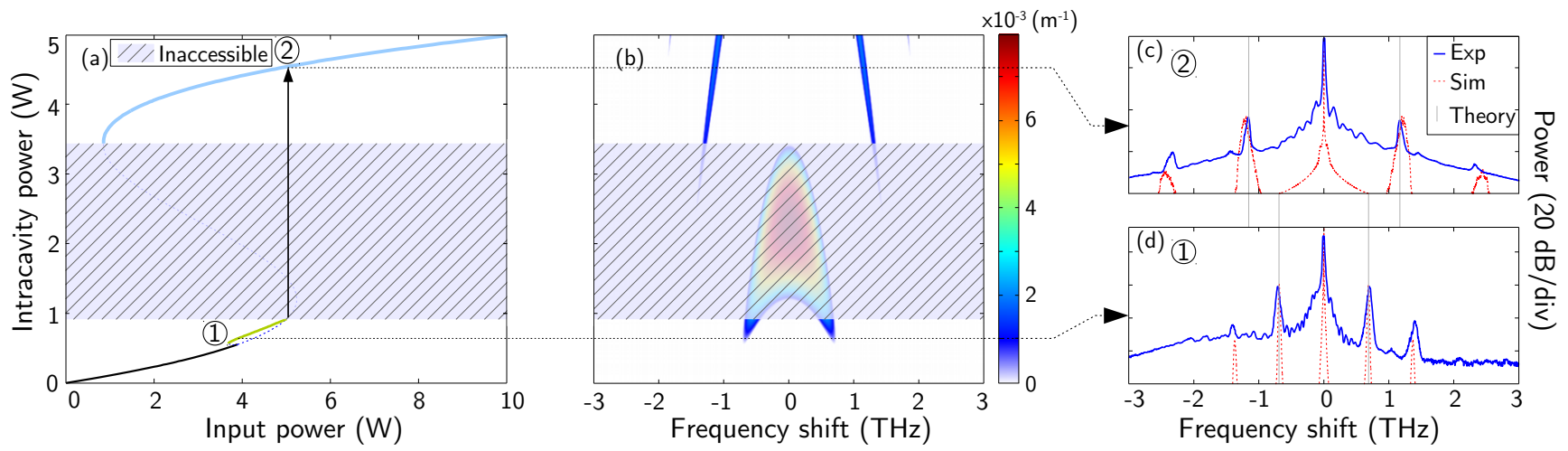

Fig. 5 (a) Bistable response of the cavity calculated for $\Delta=6.25(\delta=\pi / 3.2 \mathrm{rad}, \alpha=0.157)$; the hatched region is inaccessible. (b) Pseudo-color level plot of the gain spectrum as a function of the intracavity power calculated from Floquet analysis [24]. (c-d) Comparison of experimental spectra (solid blue), corresponding numerical integrations of the periodic LLE (13) (dashed red) and analytical estimates (vertical line, Theory) for two different input powers labelled 1 and 2 on the lower and upper branch respectively (see also Fig. $3(\mathrm{~d})$ ): (c) $P_{i n}=5.02 \mathrm{~W}\left(4.27 \mathrm{~W}\right.$ in experiment); (d) $P_{i n}=3.9 \mathrm{~W}(3.55 \mathrm{~W}$ in experiment). Estimated frequencies (Theory) are from Eq. (22) with $m=1$ in (c) and from Eq. (18) in (d).

of the cavity is slightly different $(49.3 \mathrm{~m}$ in the present case vs. $51.6 \mathrm{~m}$ before).

We remind that the DFT technique basically consists in stretching the temporal pulses by a highly and purely dispersive element. Note that sufficient stretching is all the easier achieved when the pump pulses are short and this is why we used shorter pump pulses. In our setup the frequency-to-time conversion is realized by a DCF with a coefficient $\beta_{2}^{D C F} \times L$ of $888 \mathrm{ps}^{2}$. The time-to-frequency mapping is then easily obtained at first order by the relation $T(\omega)=\beta_{2}^{D C F} \times L \times\left(\omega-\omega_{0}\right)$, where $\omega_{0}$ is the angular frequency of the pump. We use a fast photodiode and a $6 \mathrm{GHz}$ oscilloscope for detection and we estimate the spectral resolution to be $\approx 250 \mathrm{pm}[48]$. In order to avoid saturation of the photodetector and to be able to reveal weak MI sidelobes, the pump is filtered out using a notch filter (BSF) centered at the pump wavelength. The fiber cavity (highlighted in green in Fig. 6(a)) still exhibits normal average dispersion. The accuracy of the frequency-to-timeto-frequency conversion has been checked by comparing the results of the DFT technique to an OSA trace. Driving the resonator with a continuous pulse train of peak power $13 W$ and normalized detuning $\Delta=9$, which is large enough to allow for the two regimes of instability to be independently excited as can be seen in Fig. 3 (d). Figure 6(c) shows an OSA trace along with the superposition of 1,500 consecutive spectra acquired using the DFT setup and the corresponding averaged spectrum. A good agreement between the two detection methods is obtained for the range of frequency of interest which validates our setup.

We then proceeded to investigate the onset of the Turing and Faraday sideband growth by launching a burst of pump pulses with constant power. Working at
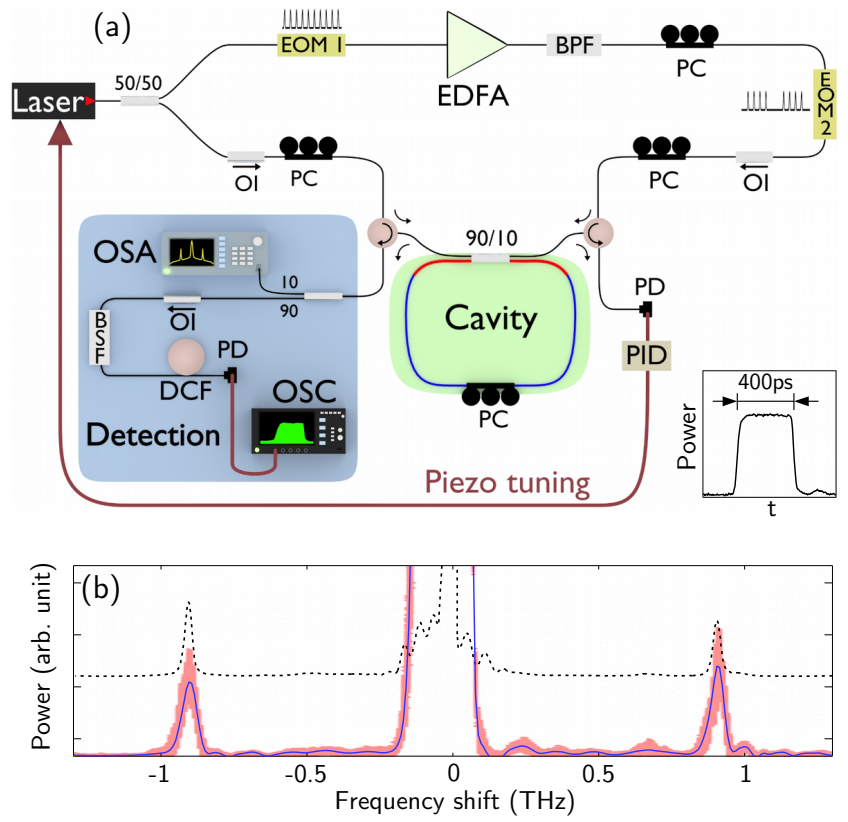

Fig. 6 (a) Experimental setup for the recording of both averaged (OSA) and round-trip-to-round-trip (OSC) spectra. Inset: profile of the pump pulses. (b) Spectrum recorded at the output of the cavity. A trace from a standard optical spectrum analyzer is in dashed black line, 1,500 consecutive traces from the DFT technique are in redwith the corresponding average spectrum superimposed in blue. The spectrum from the OSA is upshifted for the sake of clarity. EOM: electro-optic modulator; EDFA: erbium doped fiber amplifier; BPF: band-pass filter; OI: optical isolator; PC: polarization controller; PD: photo-detector; PID: proportional-integralderivative controller; BSF: band-stop filter; DCF: dispersion compensating fiber; OSA: optical spectrum analyser; OSC: oscilloscope. 


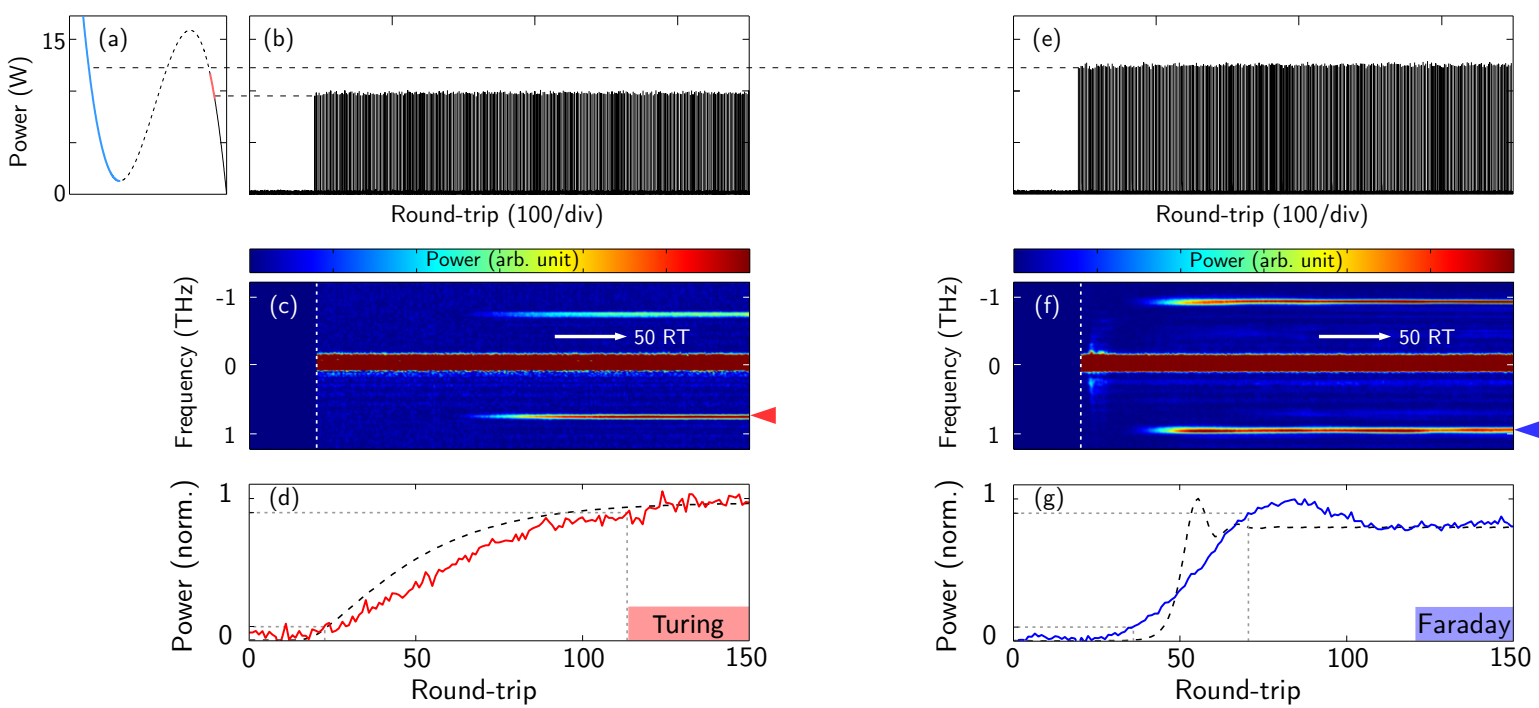

Fig. 7 (a) Bistable cycle. (b) Input pulse burst resulting in the excitation of Turing instabilities. (c) Experimental roundtrip-to-round-trip evolution of the spectrum. The dashed line indicates the first input pulse. The Turing sideband position is marked by a red arrow on the right. (d) Evolution of the power contained in the unstable sideband indicated by the colored triangle. Numerical simulation of this transition is superimposed in dashed black line. Dashed gray lines indicate 10 and $90 \%$ of the rise time. (e-g) Same as (b-d) but for an input peak power of $13 \mathrm{~W}$. This results in the excitation of Faraday instabilities on the upper branch of the bistable cycle.

fixed detuning, the nature of the instability observed only depends on the peak power of the pump pulses: in our setting, $10 \mathrm{~W}$ for the Turing branch and $13 \mathrm{~W}$ for the Faraday branch (Fig. 7(a) and (d respectively, where bistable cycles are shown). Figure 7(b) and (e) show that in both cases a pair of spectral sidebands appear symetrically to the pump after a few round-trips, at a frequency shift slightly higher for Faraday sidebands, which is in agreement with theory and previous experiments $[24,29]$. Furthermore this allows us to unambiguously identify the observed regime of instability, as shown in the previous section. The growth of the power of the unstable modes eventually saturates as the system reaches a stable attractor of the dynamics [35]. This is clearly illustrated by Fig. 7(c) and (f) which showcase the temporal evolution of the power content of the high-frequency sideband in the two regimes. By comparing Fig. 7(c) and (f), one clearly notices that the transition to the stable Turing regime appears to be slower than the transition to the Faraday regime. A simple and intuitive explanation lies on the difference of parametric gain between the two regimes of instability. Indeed, we verified that the parametric gain is more than two times higher in the Faraday case compared to the Turing case. On the other hand, the rise time of the Faraday mode is nearly two times shorter than the one of the Turing mode, as can be seen in Fig. 7(c) and (f). This supports the argument that the difference in the dynamics between these two regimes is essentially driven by the strength of the parametric gain. In order to compare with the experimental recordings, we also conducted numerical simulations based on the LLE (12) seeded at the unstable frequency. The outcome is displayed as dashed black lines in Fig. 7(c) and (f). A good qualitative agreement is obtained, clearly highlighting the faster transition in the case of the Faraday instability.

Besides the emergence of the Faraday unstable branch in addition to the Turing one, the inhomogeneous dispersion profile allows the transition between these instability regimes by simply tuning the input power while keeping the detuning fixed. This power dependence has been observed in the stationary regime through the abrupt change of unstable frequency associated to the two instabilities [29]. Here we address the question of the transition between those instabilities by recording the real-time evolution of the cavity output spectrum when abruptly tuning the input pump power from a level corresponding to the Turing regime to a higher one which corresponds to the Faraday regime and vice versa.

Figure 8(b) shows the evolution of the output spectrum corresponding to the pump power evolution of Fig. 8(a). The abrupt power step is preceded by a large number of pump pulses of $10 \mathrm{~W}$ peak power (not shown here) to ensure that a stable Turing state is reached. Once again, the different regimes of instability can be identified owing to their different characteristic frequencies. The sudden increase of pump power appears to trigger the Turing-to-Faraday transition i. e. the switch- 

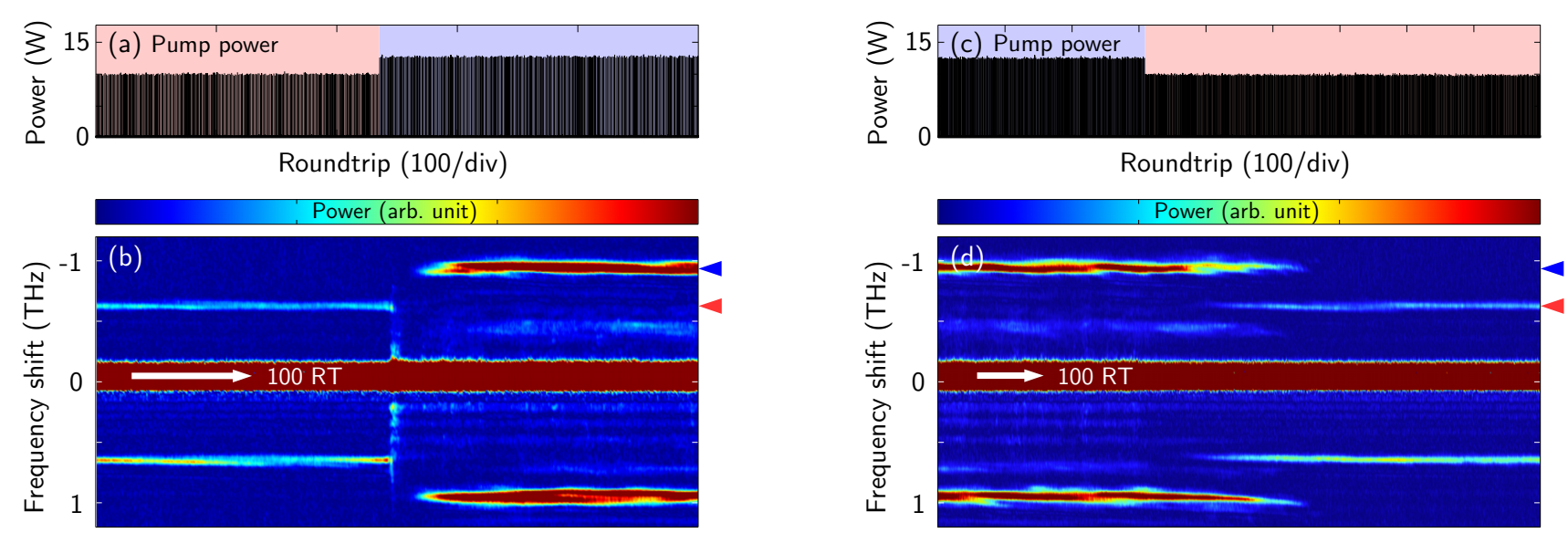

Fig. 8 (a) Input pulse pattern exhibiting an abrupt increase of the pump power. The two levels correspond to peak pump powers of 10 and $13 \mathrm{~W}$. (b) Corresponding experimental evolution of the cavity output spectrum showing the Turing-toFaraday transition. Turing (Faraday) sideband's position is marked by a red (blue) arrow on the right. (c-d) Same as (a-b) but for a sudden decrease of the input power, thus triggering the Faraday-to-Turing transition.

ing of the system from the lower branch to the upper branch of the bistable cycle. The opposite transition can occur when we switch back the input power to its lower value. This is illustrated in Figs. 8(c-d) which shows that, after such modification of the driving field, the system goes back to a stationary state on the lower branch of the bistable cycle in the region of Turing instability. To summarize, we showed that the dynamics of dispersion oscillating cavities can be experimentally investigated in details by means of a standard DFT technic which allows the round to round trip recording of output spectra. It revealed that by simply tuning the pump power one can trigger Turing and Faraday instabilities.

\section{Conclusion}

We investigated theoretically and experimentally the development of different kinds of instabilities in passive ring cavities composed of pieces of fiber with different dispersion. We presented an original derivation of the well known Lugiato-Lefever equation adapted for the description of dispersion oscillating cavity. Quite remarkably, we demonstrated that this equation is valid well beyond the mean field approximation, under which it has been traditionally derived. We reviewed the theory of Turing (modulational) and Faraday (parametric) instability in inhomogeneous fiber cavities by means of Floquet theory. We reported the experimental demonstration of the generation of stable Turing and Faraday patterns in the same device, which can be controlled by changing the detuning and/or input power. Moreover, we experimentally recorded the round-tripto-round-trip dynamics of the spectrum by implement- ing a real-time spectroscopy technique. We found that Turing and Faraday instabilities not only differ by their characteristic frequency but also by their dynamical behavior. In more general terms, we showed that compared to other experimental setups fiber ring cavities made of dispersion oscillating fibers are a fantastic test bed for fundamental investigations since the dynamics can easily be recorded by means of simple DFT technics and the dispersion step easily tuned with different optical fibers. It paves the way to more complete experimental and fundamental investigations of parametric instabilities in passive resonators, namely of their nonlinear regimes. Besides, these fundamental investigations can find direct applications in the context of soliton or frequency comb generation in microresonators.

Acknowledgements This work was partly supported by IRCICA (USR 3380 Univ. Lille - CNRS), by the Agence Nationale de la Recherche (grants TOPWAVE, NoAWE, Labex CEMPI and Equipex FLUX), by the French Ministry of Higher Education and Research, the Nord-Pas de Calais Regional Council and Fonds Européen de Développement Économique Régional (grant CPER Photonics for Society). S.T. acknowledges also the grant PRIN 2012BFNWZ2. We are grateful to Laure Lago for providing the fiber Bragg gratings.

\section{References}

1. G. Van Simaeys, P. Emplit, M. Haelterman, Phys. Rev. Lett. 87(3), 033902 (2001)

2. G. Van Simaeys, P. Emplit, M. Haelterman, J. Opt. Soc. Am. B 19(3), 477 (2002)

3. B. Kibler, J. Fatome, C. Finot, G. Millot, F. Dias, G. Genty, N. Akhmediev, J.M. Dudley, Nat Phys 6(10), $790(2010)$

4. B. Kibler, J. Fatome, C. Finot, G. Millot, G. Genty, B. Wetzel, N. Akhmediev, F. Dias, J.M. Dudley, Scientific Reports 2 (2012) 
5. J.M. Dudley, G. Genty, S. Coen, Rev. Mod. Phys. 78(4), 1135 (2006)

6. J.M. Dudley, J. Taylor, Supercontinuum Generation in Optical Fibers, 1st edn. (Cambridge University Press, 2010)

7. D.R. Solli, C. Ropers, P. Koonath, B. Jalali, Nature 450(7172), 1054 (2007)

8. N. Akhmediev, B. Kibler, F. Baronio, M. Beli, W.P. Zhong, Y. Zhang, Wonkeun Chang, J.M. Soto-Crespo, P. Vouzas, P. Grelu, C. Lecaplain, K. Hammani, S. Rica, A Picozzi, M. Tlidi, K. Panajotov, A. Mussot, A. Bendahmane, Pascal Szriftgiser, G. Genty, J. Dudley, A. Kudlinski, A. Demircan, U. Morgner, Shalva Amiraranashvili, C. Bree, G. Steinmeyer, C. Masoller, N.G.R. Broderick, A.F.J. Runge, Miro Erkintalo, S. Residori, U. Bortolozzo, F.T. Arecchi, S. Wabnitz, C.G. Tiofack, S. Coulibaly, M. Taki, J. Opt. 18(6), 063001 (2016)

9. P. DelHaye, A. Schliesser, O. Arcizet, T. Wilken, R. Holzwarth, T.J. Kippenberg, 450(7173), 1214

10. T. Hansson, D. Modotto, S. Wabnitz, Phys. Rev. A 88(2), 023819 (2013)

11. K. Tai, A. Hasegawa, A. Tomita, Phys. Rev. Lett. 56(2), $135(1986)$

12. M.E. Marhic, Fiber Optical Parametric Amplifiers, Oscillators and Related Devices, 1st edn. (Cambridge University Press, 2007)

13. J. Hansryd, P.A. Andrekson, M. Westlund, J. Li, P.O. Hedekvist, IEEE Journal of Selected Topics in Quantum Electronics 8(3), 506 (2002)

14. C. Caucheteur, D. Bigourd, E. Hugonnot, P. Szriftgiser, A. Kudlinski, M. Gonzalez-Herraez, A. Mussot, Optics Letters 35(11), 1786 (2010)

15. S. Radic, IEEE Journal of Selected Topics in Quantum Electronics 18(2), $670(2012)$

16. G.L. Oppo, J Math Chem 45(1), 95 (2009)

17. J.K. Jang, M. Erkintalo, S. Coen, S.G. Murdoch, Nature Communications 6, 7370 (2015)

18. S.B. Papp, K. Beha, P. DelHaye, F. Quinlan, H. Lee, K.J. Vahala, S.A. Diddams, Optica, OPTICA 1(1), 10 (2014)

19. V. Brasch, E. Lucas, J.D. Jost, M. Geiselmann, T.J. Kippenberg, Light Sci Appl. 6(1), e16202 (2017)

20. M. Droques, A. Kudlinski, G. Bouwmans, G. Martinelli, A. Mussot, Optics Letters 37(23), 4832 (2012)

21. F. Matera, A. Mecozzi, M. Romagnoli, M. Settembre, Opt. Lett. 18(18), 1499 (1993)

22. C. Finot, J. Fatome, A. Sysoliatin, A. Kosolapov, S. Wabnitz, Opt. Lett. 38(24), 5361 (2013)

23. A. Armaroli, F. Biancalana, Opt. Express 20(22), 25096 (2012)

24. M. Conforti, A. Mussot, A. Kudlinski, S. Trillo, Opt. Lett. 39(14), 4200 (2014)

25. K. Staliunas, C. Hang, V.V. Konotop, Phys. Rev. A $\mathbf{8 8}(2), 023846(2013)$

26. A. Perego, N. Tarasov, D. Churkin, S. Turitsyn, K. Staliunas, Phys. Rev. Lett. 116(2), 028701 (2016)

27. A. Nayfeh, D. Mook, Nonlinear Oscillations. Wiley Classics Library (Wiley, 2008)

28. M. Faraday, Philosophical Transactions of the Royal Society of London (January), 379 (1931)

29. F. Copie, M. Conforti, A. Kudlinski, A. Mussot, S. Trillo, Phys. Rev. Lett. 116(14), 143901 (2016)

30. F. Copie, M. Conforti, A. Kudlinski, S. Trillo, A. Mussot, Opt. Lett., OL 42(3), 435 (2017)

31. M. Conforti, F. Copie, A. Mussot, A. Kudlinski, S. Trillo, Opt. Lett., OL 41(21), 5027 (2016)

32. L.A. Lugiato, R. Lefever, Phys. Rev. Lett. 58(21), 2209 (1987)
33. M. Haelterman, S. Trillo, S. Wabnitz, Optics Communications 91(56), 401 (1992)

34. K. Ikeda, O. Akimoto,

35. M. Haelterman, S. Trillo, S. Wabnitz, Opt. Lett. 17(10), $745(1992)$

36. M. Conforti, A. Mussot, A. Kudlinski, S. Trillo, Optics letters (14), 4200 (2014)

37. M. Anderson, Y. Wang, F. Leo, S. Coen M. Erkintalo, S. Murdoch, (2017). URL http://arxiv.org/abs/1702.00782

38. S. Coen, M. Haelterman, Opt. Lett. 24(2), 80 (1999)

39. S.R. Nodari, M. Conforti, G. Dujardin, A. Kudlinski, A. Mussot, S. Trillo, S. De Bièvre, Physical Review A 92(1), 013810 (2015)

40. S. Coen, M. Haelterman, P. Emplit, L. Delage, L.M. Simohamed, F. Reynaud, J. Opt. B: Quantum Semiclass. Opt. 1(1), 36 (1999)

41. X. Wang, D. Bigourd, A. Kudlinski, K.K.Y. Wong, M. Douay, L. Bigot, A. Lerouge, Y. Quiquempois, A. Mussot, Opt. Lett., OL 39(7), 1881 (2014)

42. D.R. Solli, G. Herink, B. Jalali, C. Ropers, Nat Photon 6(7), $463(2012)$

43. B. Wetzel, A. Stefani, L. Larger, P.A. Lacourt, J.M. Merolla, T. Sylvestre, A. Kudlinski, A. Mussot, G. Genty, F. Dias, J.M. Dudley, Scientific Reports 2, 882 (2012)

44. M.J. Schmidberger, D. Novoa, F. Biancalana, P.S.J. Russell, N.Y. Joly, Opt. Express, OE 22(3), 3045 (2014)

45. A.F.J. Runge, N.G.R. Broderick, M. Erkintalo, Journal of the Optical Society of America B: Optical Physics 33(1), 46 (2016)

46. J. Hammer, P. Hosseini, C.R. Menyuk, P.S.J. Russell, N.Y. Joly, Opt. Lett., OL 41(20), 4641 (2016)

47. G. Herink, B. Jalali, C. Ropers, D.R. Solli, Nat Photon 10(5), 321 (2016)

48. K. Goda, B. Jalali, Nat Photon 7(2), 102 (2013) 